Rabaska

Revue d'ethnologie de l'Amérique française

\title{
Les Mémoires du père Anselme Chiasson
}

\section{Ronald Labelle}

Volume 2, 2004

URI : https://id.erudit.org/iderudit/201650ar

DOI : https://doi.org/10.7202/201650ar

Aller au sommaire du numéro

Éditeur(s)

Société québécoise d'ethnologie

ISSN

1703-7433 (imprimé)

1916-7350 (numérique)

Découvrir la revue

Citer ce document

Labelle, R. (2004). Les Mémoires du père Anselme Chiasson. Rabaska, 2,

123-148. https://doi.org/10.7202/201650ar d'utilisation que vous pouvez consulter en ligne.

https://apropos.erudit.org/fr/usagers/politique-dutilisation/ 


\section{Les Mémoires du père Anselme Chiasson}

Le texte qui suit est un condensé des Mémoires inédits du père Anselme Chiasson, rédigés en 2002 et déposés au Centre d'études acadiennes. J'ai tenté de conserver l'essentiel du texte, tout en me contraignant aux exigences de la revue en ce qui concerne la longueur. Si certains sujets semblent manquer de développement, il faut se rappeler que le présent texte est une collection d'extraits rassemblés dans le but de présenter un portrait du père Anselme Chiasson, et non une biographie complète. Je remercie le père Anselme de $m$ 'avoir permis de retenir tels quels dans ses Mémoires ceux de ses souvenirs qui nous en apprennent beaucoup, autant sur ce grand personnage que sur son époque.

RoNALD LABELLE

\section{Extrait de l'avant-propos par le père Anselme Chiasson}

J'ai écrit ces mémoires pour m'amuser et pour revivre des souvenirs du passé en me les remémorant autant que possible et en tâchant de les écrire un peu par ordre chronologique. Avec cette intention en tête, je n'étais pas obligé de tout dire ni d'être trop sérieux. Ce genre permet de décrire des modes de vie et certains événements, ainsi que diverses manières d'agir d'une époque révolue. Qu'on sache bien aussi que ce texte a été écrit en grande partie de mémoire...

Enfin, ayant publié plusieurs volumes, je ne doute pas qu'un jour des étudiants de l'Université, ou d'autres, après Marguerite Maillet ${ }^{1}$, entreprendront de faire des études sur la carrière de nos auteurs. S'ils veulent connaître la mienne, ils n'auront qu'à compulser mes archives, puiser dans ces mémoires et s'amuser eux aussi.

1. Auteur de plusieurs ouvrages de référence sur la littérature acadienne. 


\section{MON ENFANCE}

Je suis né en pleine nuit, en plein hiver et en pleine tempête, un mardi, le 3 janvier 1911, au Petit-Étang, un district de Chéticamp au Cap-Breton, en Nouvelle-Écosse. Mon père était Thimothée Chiasson ${ }^{2}$ et ma mère Colombe Boudreau ${ }^{3}$. Au baptême, je reçus le nom de Charles et on m'appelait Charlie. Le nom d'Anselme, que je porte et par lequel je suis connu, me fut donné à ma prise d'habit religieux, au début de mon noviciat chez les Capucins.

J'étais le quatrième d'une famille de neuf enfants. Vu qu'un autre enfant m'a suivi à moins d'un an et demi de distance, je n'ai pas dû me faire gâter bien longtemps. Je me souviens que vers l'âge de six ans, j'ai attrapé une pneumonie qui a manqué m'emporter. Le père LeBlanc est venu me donner le sacrement des malades. Il m'avait parlé du ciel et m'avait demandé si j'acceptais d'aller voir Jésus. Et j'avais dit oui. J'étais décompté, comme on disait; mais le docteur William LeBlanc m'a sauvé.

Le soir, notre maison était souvent le lieu de rassemblement des personnes âgées du voisinage, surtout quand mes grand-père et grand-mère maternels, qui demeuraient au havre, venaient se promener chez nous pour quelques jours. Durant ces veillées, on parlait des fondateurs acadiens de Chéticamp, de leur façon de vivre; on racontait des légendes et des contes, dont quelquesuns étaient effrayants pour de jeunes oreilles comme les nôtres. Nos parents ne se rendaient pas compte de l'influence de ces histoires sur nos jeunes imaginations. Moi, $j$ 'en fus marqué. Ces soirées, si elles créèrent un traumatisme passager chez l'enfant que j'étais, eurent surtout des effets bénéfiques.

Ces bons vieillards, bien qu'illettrés, jouissaient d'une mémoire phénoménale. Petits-fils des déportés de 1755 , que de détails ils savaient raconter sur les drames qu'avaient eu à subir leurs ancêtres ! Combien colorés leurs récits sur la vie des pionniers de Chéticamp : la chasse à l'orignal ou au phoque, leurs relations amicales avec les Micmacs de passage à qui ils donnaient une hospitalité toute fraternelle, les miracles des anciens curés, les exploits des hommes forts, les légendes auxquelles ils croyaient. Ces histoires merveilleusement bien racontées me passionnaient au point qu'à ma première année de collège en 1927, à 16 ans, je pris la résolution d'écrire un jour la monographie paroissiale de ma place natale qui, tout en fournissant les dates et les informations historiques, décrirait aussi cette vie des gens de chez nous.

2. Thimothée à Lubin, à Thimothée à Thomas à Basile, à Paul, à Jacques, à Sébastien, à Guyon venu de La Rochelle, France, et fils de Pierre Chiasson et de Marie Pérocher.

3. Colombe à Charles, à Venant, à Charles, à Joseph, à Germain, à François, à Charles, à Michel Boudreau venu aussi de la Rochelle. 
J'en traçai même la table de matière à laquelle je suis demeuré étonnamment fidèle dans mon livre Chéticamp, histoire et traditions acadiennes, publié en 1961.

\section{L'âge scolaire}

Je me rappelle vaguement ma première journée à l'école. J'y fus certainement amené par un de mes grands frères ; donc pas trop de problèmes. De mon séjour à la petite école de notre coin, au Petit-Étang, je garde davantage le souvenir des jeux auxquels je participais durant les récréations que des classes elles-mêmes. Je me souviens de ma première ardoise, toute neuve et bien nette dans son cadre de bois, de mes premiers livres aussi tout neufs et qui sentaient bon.

L'instruction n'était pas toujours bien vue par beaucoup d'Acadiens illettrés. «Tu veux vivre du crayon, en paresseux !» me répétait un voisin. Ma mère était plus intelligente que ça et, au contraire de tant d'autres, nous encourageait à étudier. Elle veillait à ce qu'on apprenne fidèlement nos leçons et qu'on fasse nos devoirs. L'hiver, on étudiait autour de la table à manger, à la lumière si faible d'une lampe à parafine $\mathrm{e}^{4}$ qu'on se demande comment on pouvait faire pour ne pas s'arracher les yeux. Les classes étaient mixtes à Chéticamp, même à l'école du couvent, les garçons d'un bord et les filles de l'autre. Je n'y ai jamais vu de problèmes, au contraire. Il se créait une saine émulation entre les sexes. J'arrivais ordinairement bon premier aux examens ; mais je me suis particulièrement appliqué à maintenir ce but quand une compagne de classe, Dorothée Chiasson, me talonnait fort pour cette première place. À cette époque, on n'était pas tellement protégé contre l'arbitraire ou les caprices des maîtres. Une fois, alors qu'on était en rang pour un exercice de lecture à haute voix, le maître qui était mon oncle se tenait derrière moi et me tirait, à l'arracher, une couette de cheveux de mon

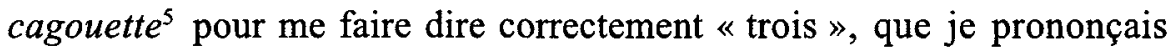
« tras ». Il me faisait si mal que je m'étais promis de lui donner une raclée quand je serais grand.

À notre petite école, tous les élèves allaient nu-pieds une partie de l'année, c'est-à-dire en juin et en septembre. Le reste du temps, nous chaussions des souliers et des hausses de peau de bœuf fabriqués à la maison. Notre butin était d'étoffe foulée ${ }^{6}$, sauf les bas, les mitaines et les calottes que nos mères brochaient $t^{7}$ en laine. À la maison comme à l'école, point d'électricité ni d'eau courante. Un poêle à bois servait à cuire les aliments et à réchauffer la

4. Huile de pétrole.

5. Derrière de la tête, nuque.

6. Pour plus de détails sur notre linge, nos jeux et notre vie d'alors, voir mon livre Chéticamp, histoire et traditions acadiennes, Moncton, Éditions des Aboiteaux, 1961 (1962, 1972).

7. Tricoter. 
maison. Aucune isolation au toit. Aussi, on voyait au grenier les clous qui traversaient la toiture devenir tout blancs l'hiver, se grossir de givre et péter comme des coups de fusil sous l'action du froid. On couchait deux ou trois dans le même lit ou la même couchette, sous plusieurs couvertes ${ }^{8}$ avec une calotte de laine sur la tête. Le matin notre père se levait avant nous pour allumer le poêle et réchauffer la maison.

Nous avions de bons parents. Papa nous aimait certainement, mais nous ne nous sentions pas proches de lui comme de maman. Je me suis toujours senti aimé et considéré de la part de maman. J'ai l'impression qu'elle me portait une attention particulière. Elle me parlait, me donnait des conseils avec une grande douceur maternelle. Elle me faisait lire des livres choisis et me dirigea très jeune à l'école du couvent. Pressentait-elle en moi une vocation sacerdotale, comme les religieuses plus tard ? J'ai l'impression que oui.

La maison paternelle était bordée de deux côtés par des bosquets d'épinettes. Que de mystères recelait pour des petits gars comme nous ce qui nous apparaissait comme d'immenses forêts ! Endroit idéal pour jouer à cachecache ou aux Indiens. Des petits sentiers que nous étions seuls à connaître nous conduisaient à des éclaircies où se jouaient les rayons de soleil, ou à des sous-bois obscurs. L'hiver, c'était la neige entre les arbres qu'amoncelaient les fameux suetes ${ }^{9}$. Nous y découvrions parfois des lièvres, des perdrix ou des chats étrangers. Nous faisions la chasse aux uns et aux autres. Mais les oiseaux qui y faisaient leurs nids étaient bien protégés, car nous étions persuadés qu'il était péché de les tuer. Tout au plus grimpions-nous dans les arbres pour admirer leurs œufs ou leurs petits. À un tiers de mille, en arrière de chez nous, coulait un cours d'eau appelé la Petite-Rivière. Nous allions y patiner l'hiver, nous y baigner l'été et y pêcher la truite et l'anguille. Un ruisseau s'y jetait, bordé d'immenses prairies que sillonnaient de nombreux rats musqués. J'en prenais au piège et leur levais la peau que j'entrais à l'envers sur un moule pour la faire sécher, puis la vendre.

Le coin de Chéticamp habité par nos familles de Chiasson, grand-père Lubin, son frère Placide, Lubin à Luc, ma tante Tarsile, s'appelait Les Caves. En effet, ces gens étaient logés le long d'une suite de cinq ou six immenses caves naturelles de trente mètres de profondeur et un peu plus de largeur, peuplées d'arbres, avec un lac au fond de chacune. L'hiver, les enfants, nous avions là nos pentes naturelles de glissade. Une année, notre voisin, Lazare (à Alexandre) Poirier, m'avait fabriqué un magnifique traîneau. Ce fut tout un cadeau pour le petit gars que j'étais $!^{10}$ Nous vivions en pleine campagne et en pleine nature, avec la mer toujours en face de nous et les montagnes en

8. Couvertures de lit.

9. Vents terribles du sud-est.

10.Mon frère Wellie-Mose dit que c'est pour lui que Lazare le fit. Peut-être a-t-il raison. 
arrière. Ces vastes paysages m'ont marqué et, ayant à vivre ailleurs, j'en ai eu la nostalgie et m'en suis ennuyé toute ma vie.

Rendu à la septième année à la petite école, je fus transféré par mes parents à l'école plus haut cotée du couvent, au havre, à cinq kilomètres de chez nous. Je parcourais ce trajet matin et soir durant la belle saison ; en chemin, pendant quelques mois, je subis les vexations d'élèves plus costauds. Durant l'hiver je restais au havre chez mon grand-père maternel, Charles (à Venant) Boudreau. J'y mangeais et couchais durant la semaine, mais retournais chez nous le vendredi soir. En compensation, mes parents fournissaient pour ma pension un demi-sac de farine par hiver.

Les religieuses enseignantes, les Filles de Jésus, tenaient alors un pensionnat avec des élèves de différentes parties du Cap-Breton, dont plusieurs unilingues anglais. Ces pensionnaires suivaient les mêmes classes que nous, ce qui entraînait un usage presque exclusif de la langue anglaise dans l'enseignement. D'ailleurs, tous les manuels étaient en anglais, même la grammaire française. La première année, j'eus beaucoup de difficultés à cause de l'anglais. Si je pouvais lire l'anglais et comprendre un peu ce que je lisais, je n'entendais rien au parler de cette langue. Un jour, notre institutrice, sœur Sainte-Hughes qui pouvait faire des colères terribles, en fit une devant la classe. Ne comprenant rien à ce qu'elle disait, je me penche vers mon compagnon et lui demande : "Contre qui crie-t-elle ? - C'est contre toi »! Avec le souvenir que j'ai de cet élève, je doute fort que ce fût vrai.

J'étais plutôt timide et sensible de nature. Aussi, je ne me rappelle pas de m'être battu une seule fois, comme cela arrive entre écoliers. Ce n'est pas que j'étais peureux ou feluette ${ }^{11}$, non. Je n'aimais pas la chicane, tout simplement. Lorsque deux élèves se battaient, alors que les autres les entouraient, moi je fuyais la scène. Je n'ai jamais dansé. Je suis parti de Chéticamp à dix-sept ans. À cet âge, plusieurs de mes compagnons se joignaient aux plus âgés pour danser des sept ou des huit aux bras d'une compagne. J'ai assisté à quelques noces, sans jamais prendre part à la danse. Ces danses duraient toute la nuit au son du violon. Et quand on repartait le matin, ordinairement en carriole, puisque les mariages avaient surtout lieu en hiver, on avait la tête, le cour, tout l'être, remplis de reels entendus toute la nuit, et tous les bruits en reprenaient le rythme, le choc des sabots du cheval, le crissement des patins de la carriole sur la neige, et surtout les grelots.

11. Fluet. 


\section{Décès de ma mère et ses suites}

En 1925, à l'âge de 14 ans, j'ai perdu ma mère, morte d'une pneumonie. Elle était âgée de 45 ans seulement et laissait mon père avec six enfants à la maison, dont cinq garçons et une petite fille de quatre ans. Elle fut exposée à notre demeure deux jours et deux nuits. Tous ceux et celles qui vinrent prier au corps, et ils furent nombreux, pleuraient devant le spectacle d'une famille frappée si douloureusement et en désarroi complet. Souvent, dans de tels cas, le père donnait ses enfants, un à un, à des familles qui s'offraient à les adopter. Mon père ne pouvait se décider à un tel geste de séparation. Heureusement, une de ses sœurs, ma tante Olive, était demeurée célibataire pour garder toute une maisonnée, son père et sa mère, sa grand-mère, son frère handicapé et un neveu qu'elle avait adopté quand il était bébé. La maison était déjà pleine. Or, dans un geste d'une générosité incroyable, elle invita mon père à déménager chez elle avec toute sa famille. Quant à moi, vu que durant la semaine je pensionnais déjà chez mon grand-père au havre, celui-ci m'offrit d'y demeurer en permanence. Ce que je fis.

J'allais souvent visiter ma famille chez ma tante Olive. La maison n'était point grande et ils y vivaient tassés les uns sur les autres comme des sardines. Or, je n'ai jamais entendu une plainte de la part des grands-parents, ni de tante Olive, ni de Loubie qui avait déjà quinze ans et qu'on devait déranger énormément. Mon grand-père me dit même un jour : « Tu sais, si tu t'ennuies là-bas ou si tu n'es pas bien, viens-t-en rester avec nous autres. La maison est pleine, mais on peut toujours trouver une place. On s'y rencontrera su'le cant $^{12} »$.

Chez mon grand-père, au havre, je m'efforçais de rendre service. J'aidais aux semailles du printemps et aux récoltes de l'automne, à scier, à fendre et à entrer le bois de chauffage, au soin des animaux l'hiver. L'été, je conduisais les vaches au parc de pacage le matin et retournais les chercher le soir. J'étais renfermé sur moi-même et je communiquais difficilement avec les adultes, même avec la famille de mon grand-père. Par ailleurs, je partageais avec plaisir les jeux de mes nombreux cousins et cousines qui demeuraient à côté, les enfants de mon oncle Placide Boudreau.

\section{Origine de ma vocation religieuse}

Je me rappelle que lorsque $\mathrm{j}$ 'étais encore jeune, ma mère aimait lire les dimanches après-midi, quand tout était calme. Les livres étaient rares à cette époque. Mais le curé, l'abbé Patrice LeBlanc, avait monté une petite bibliothèque sous la sacristie, où les paroissiens pouvaient emprunter des livres.

12. Prononcé « can ». Signifie : de côté, de biais. 
Ma mère lisait des romans pieux ou légendaires, comme sainte Geneviève de Brabant et autres, la vie des saints, le Paroissien romain. Elle nous incitait à lire aussi. Vers dix ou onze ans, elle me fit lire la vie de saint Gérard Majella qui me marqua profondément. Cette lecture créa en moi un idéal très élevé, une piété profonde, une grande dévotion à l'Eucharistie, à la sainte Vierge. À partir de ce moment-là, après le chapelet et la prière du soir en famille, moi, privément, j'ajoutais deux autres chapelets pour compléter le rosaire. $\mathrm{Au}$ lever, agenouillé au pied de mon lit, je récitais par cœur la longue prière du matin d'alors. Chez mon grand-père Charles, on me réveillait tôt pour que j'aille mener les vaches au pacage avant de me rendre à l'école. En me levant, je récitais cette longue prière à genoux dans ma chambre. La famille, qui ignorait ça, pestait contre la lenteur, pensait-elle, que je mettais à m'habiller.

Vers le même âge, je désirais devenir prêtre. Mais à cette époque, un tel rêve était utopique. Les conditions financières de ma famille ne laissaient aucun espoir à ce sujet. Mais Méderic LeBlanc, de Chéticamp, le père Euchariste en religion, était entré chez les capucins et devenu missionnaire en Éthiopie. Il écrivait des articles dans le journal L'Évangéline, que nous recevions, et des lettres à sa famille et à des amis. Tout le monde à Chéticamp parlait de lui avec vénération. De mon côté, je priais la sainte Vierge de me mettre sur le chemin où je pourrais un jour, si je voulais, devenir moi aussi missionnaire à l'exemple du père Euchariste.

En 1927, le père Euchariste revint au pays et, à Chéticamp, il prêcha aux messes du dimanche. Il vint à l'école parler aux élèves des hautes classes de ses missions, des capucins, de la possibilité de le suivre et de devenir missionnaire comme lui. Il invita les intéressés à aller le voir dans un bureau à côté de la classe. Sœur Sainte-Agnès, notre institutrice, m'encouragea fortement à aller lui parler. J'y fus. Il avait la parole facile, presque enjôleuse. Il me parla d'abord de mon père qu'il connaissait très bien, puis du Collège séraphique des capucins à Ottawa, des bons cours qu'on y donnait, de l'esprit de famille qui y régnait, des vacances que les élèves prenaient ensemble au lac Meech, près de là, avec chaloupes et canots sauvages, dans un paysage enchanteur. Surtout, il m'offrit de défrayer lui-même toutes mes dépenses de pension et de voyages. C'était la réalisation rendue possible de mon rêve d'enfance. Et j'acceptai. Deux de mes compagnons de classe, Abraham Chiasson et son cousin Louis Chiasson, décidèrent d'y venir aussi.

Nous étions en septembre avancé. Nous partîmes avec le père Euchariste. Le bagage de chacun n'était pas lourd. Nous ignorions totalement ce qu'il fallait pour l'entrée au collège en fait d'articles de toilette ou même de linge. Je n'étais jamais sorti de Chéticamp. Je n'avais jamais vu de salle de bain, ni 
de toilette intérieure, ni d'électricité, ni les gros chars ${ }^{13}$. Quelqu'un vint nous conduire à Inverness, où nous couchâmes ce soir-là chez des amis du père Euchariste. Le lendemain, nous prenions le petit train d'Inverness pour PortHawkesbury. Pour nous, c'était les gros chars, même si les banquettes étaient en bois, si chaque wagon était chauffé avec un poêle à bois et éclairé au fanal. À Port-Hawkesbury, on changeait de train pour prendre celui de Sydney à Halifax, plus luxueux, et à Truro, celui d'Halifax à Montréal.

\section{MES ANNÉES DE COLLÈGE}

Enfin, nous arrivons à Ottawa, au Collège séraphique, comme on 1'appelait alors. Le père Euchariste n'avait pas prévenu personne de notre arrivée et les classes étaient commencées depuis plus d'un mois. Le directeur a failli ne pas nous accepter. Il n'y avait pas eu d'examen préalable, comme cela se faisait avec chaque élève qui sollicitait son entrée, pas de certificat d'études, pas de lettres de recommandation du curé ni de nos institutrices. Rien! Mais, on venait de si loin ! On accepta de nous prendre à l'essai.

Notre situation frisait le désarroi. Imaginez trois jeunes Acadiens venus de l'autre bout du monde, qui ne connaissaient rien d'autre que ce qu'ils avaient appris dans les livres, - et encore ! dans des livres anglais ! -, qui sortaient du fond d'une campagne où il n'existait rien des commodités modernes ! Il devait nous manquer beaucoup de choses comme linge et articles de toilette. En plus, nous ne parlions que la langue archaïque acadienne. Nous étions vraiment des objets de curiosité pour les autres élèves, tous Québécois ou Franco-Ontariens. Quant à nous, c'était le dépaysement complet. Heureusement, le collège était de dimension modeste, un juvénat de quatre-vingts élèves seulement. Et nous fûmes tout de suite entourés d'une sympathie chaleureuse. À Chéticamp, nous avions appris un peu de latin, étudié les mathématiques, l'algèbre, et Abraham et moi avions commencé la trigonométrie. Dans ces matières et en anglais, nous dépassions de cent coudées nos confrères du collège. On nous dispensa donc de l'anglais et des mathématiques pour nous permettre de concentrer davantage nos efforts sur la grammaire française où nous étions plus faibles.

Loin de ma famille, loin de l'Acadie, je sentais une nostalgie profonde. Il n'y a pas comme d'être éloigné ou exilé pour apprécier les siens, le chezsoi qu'on a laissé, son monde, son histoire, sa patrie. Je me suis mis à lire tous les livres de la bibliothèque qui traitaient de l'Acadie, Rameau de SaintPère, Émile Lauvrière, Pascal Poirier, Longfellow, etc.

13. Le train. 


\section{Artiste en herbe}

On disait de ma famille qu' on y naissait avec une plume à dessin sur l'oreille. J'avais donc un certain don pour cet art. À l'école du couvent, j'avais gagné plusieurs premiers prix dans ce domaine aux concours agricoles. Au collège, un autre élève, Oswald Bonin, était très doué et plus avancé que moi dans cet art. J'appris beaucoup de lui. C'est avec lui que je découvris les dessins à la plume de Gérard Morisset. Avec quel plaisir j'imitais ses paysages où figuraient de grands pins aux branches chargées de neige !

Un jour, le père Euchariste, qui était devenu curé de notre paroisse d'Ottawa, vint me trouver pour que je dessine en lettres moulées Deus meus et omnia sur un immense écusson qu'il voulait placer au-dessus du maîtreautel. Ma sotte vanité fut tout émue en apercevant mon travail exposé à la vue de milliers de gens. Jusqu'à mon sacerdoce, je fus l'artiste à qui on demandait souvent d'enluminer les couvertures d'adresses qu'on lisait aux supérieurs et de peindre les décors pour les pièces de théâtre qu'on jouait. J'aimais les études et je réussissais suffisamment bien pour que, de la versification on me fit monter en belles-lettres à Noël, et, aux examens de fin d'année, j'y suis arrivé le deuxième de la classe.

\section{La vie au collège}

Si j'aimais les études, je ne peux pas en dire autant de la vie au collège. Moi qui étais né dans un pays aux vastes horizons, je m'y sentais emprisonné. Et il y avait de quoi ! Notre cour de récréation était clôturée d'un mur solide en pierre de dix pieds de hauteur. On ne pouvait rien voir au dehors, ni en être vu. On ne sortait du collège qu'en groupe et en rang pour les promenades. Moi, cependant, j'eus le privilège de sortir seul assez souvent faire des commissions pour le père directeur. En tant que portier, je m'absentais souvent des récréations pour pouvoir répondre à la porte. Je m'installais alors dans une classe d'où, par la fenêtre, je pouvais voir les gens passer dans la rue. Je dois avouer qu'il m'arrivait souvent d'envier leur liberté.

\section{Les vacances}

Avant 1927, les élèves du Collège séraphique, comme ceux de la plupart des juvénats d'alors, n'avaient pas de vacances dans leur famille. Ils les passaient tous ensemble, avec leurs pères professeurs, au lac Meech, un lac magnifique entouré de montagnes, où les pères capucins possédaient un camp d'été. Heureusement, je n'ai pas eu à subir ce régime. Dès 1927, il fut décidé de donner des vacances dans les familles l'année suivante. Et c'était heureux. C'était le meilleur test des vocations. Des élèves ne reviendraient pas et c'était 
tant mieux ! Il valait mieux qu'ils restent chez eux que de faire de mauvais religieux. Aux trois Acadiens, parce qu'ils restaient loin, on accorda un mois.

À part notre premier voyage avec le père Euchariste par Inverness et le train, tous nos autres départs se firent par bateau, de Chéticamp à Mulgrave à l'aller, et inversement au retour. Nous arrivions à Mulgrave vers 4 heures du matin. Le bateau Kinburn nous attendait au quai. Vu que tout l'équipage était composé de gens de Chéticamp, en montant sur le bateau c'était la joie de nous sentir déjà un peu chez nous. Et ce voyage de retour sur la mer, en longeant les côtes escarpées du Cap-Breton, m'émerveillait. Je passais des heures appuyé au bastingage en contemplation devant toutes ces merveilles et la majesté de la nature. J'admirais tout, les paysages, le ciel bleu, les vagues, les goélands qui nous accompagnaient en voltigeant au-dessus de nos têtes. Ce sont ces voyages qui ont inspiré les dernières pages de mon livre sur Chéticamp où je décris ces spectacles grandioses.

\section{La vocation et le noviciat}

La vocation religieuse ne se dessine pas toujours clairement et définitivement d'un coup. Elle ne se réalise pas non plus sans hésitation et chez certains sans déchirements. $\AA$ chacune de mes vacances chez nous, je prenais la résolution d'abandonner. Je le laissais entendre à ma famille et à mes amis. Mais quand arrivait la fin des vacances, une force étrange me poussait à retourner et je partais en vitesse sans en informer personne d'autre que les miens.

Je fus confirmé plus fermement dans ma vocation en rhétorique. Mon frère cadet, qui portait le nom d'Anselme, était affligé d'une maladie qui alors ne pardonnait pas, la tuberculose. Il m'écrivait régulièrement. Cette année-là, dans une lettre, sa dernière, il m'apprit qu'il avait offert sa vie pour ma vocation. Il est décédé peu de temps après. Si en plus de l'esprit de foi, d'aucuns entraient au noviciat par attrait sentimental, ce n'était pas mon cas. Comme d'autres sans doute, j'entrais par pur esprit de foi, sans attrait humain, pour ne pas être lâche devant l'appel divin que je percevais assez clairement. J'étais de ceux pour qui répondre à la vocation religieuse n'était pas facile. Seules la foi et l'aide de la grâce peuvent triompher dans de telles circonstances de nos tendances naturelles. Mais la foi et la grâce n'enlèvent pas les luttes internes. Et cet état d'âme demeura latent en moi durant tout mon noviciat. Les gens qui pensent qu'on embrasse la vie religieuse ou sacerdotale parce que c'est une vie facile se trompent. Au fond, cette lutte se poursuit toute la vie.

Malgré tout, mon noviciat ne fut pas trop difficile. La prise d'habit eut lieu le 4 juillet 1931. C'est à ce moment qu'on nous imposait notre nom de religieux. Et comme il ne fallait pas que deux religieux eussent le même nom 
dans notre province religieuse, ni qu'on porte notre propre nom de baptême, quelques religieux portaient déjà des noms assez bizarres, tels père Calasanz, père Euchariste. On nous permettait tout de même de suggérer trois noms parmi lesquels le père maître pouvait choisir, sans y être obligé cependant. Moi, je n'en avais soumis qu'un, Anselme. Personne ne portait ce nom-là dans la province. J'expliquai les raisons de mon choix : mon oncle chez qui $\mathrm{j}$ 'avais demeuré après le décès de ma mère se nommait Anselme et mon frère cadet, qui avait offert sa vie pour ma vocation, portait aussi ce nom. Je le reçus et l'ai gardé, même après le Concile Vatican II, quand on nous a permis de reprendre notre nom de baptême.

Nous étions sept novices clercs et peut-être autant de novices convers. La vie réglementée du collège nous avait déjà préparés à celle du noviciat. Couchés à neuf heures ou neuf heures et demie du soir, toute la communauté, les novices compris, était réveillée à minuit par un crécelle qu'un novice passait dans les corridors. On se rendait au chœur des religieux réciter matines et laudes du bréviaire. On se recouchait pour être réveillé à cinq heures moins quart du matin. Après notre toilette terminée, on se rendait de nouveau au chœur réciter une autre partie du bréviaire, c'est-à-dire prime et tierce, suivie d'une heure d'oraison dans l'obscurité la plus complète, et de la messe.

Nous sortions en promenade en groupe, une fois par semaine, et naturellement à pied. Comme le noviciat était situé à Limoilou, dans la basse ville de Québec, nos sorties exigeaient que nous passions par les rues de la ville. Nous portions des sandales aux pieds, les cheveux coupés en couronne autour de la tête, une jeune barbe au menton, et un habit religieux rapiécé. Comme spectacle hirsute c'était réussi. Aussi, les touristes s'arrêtaient-ils et sortaient leurs appareils de photos pour nous capter sur leurs pellicules.

\section{À notre couvent d'études}

Le noviciat terminé et nos vœux simples prononcés le 14 juillet 1932 pour trois ans, les novices clercs, qui se destinaient au sacerdoce, se rendaient à notre couvent de Pointe-aux-Trembles, la Chapelle de la Réparation, pour y poursuivre leurs études, trois ans de philosophie et quatre de théologie. Le couvent de la Chapelle de la Réparation contenait une trentaine d'étudiants, les profès simples en philosophie et les profès solennels en théologie. La vie était aussi austère. Lever à minuit, comme au noviciat, pour l'office au chœur. Lever de nouveau à cinq heures moins quart le matin. Longues heures de prière et d'oraison au chœur. Jeûne et abstinence la moitié de l'année : trois carêmes de quarante jours, tous les vendredis, la veille des fêtes de nos nombreux saints et bienheureux capucins et de la sainte Vierge où, en plus, les jeunes, nous dînions à genoux. 
Nous allions nu-pieds dans des sandales en tout temps à l'intérieur, tôt le printemps et tard l'automne à l'extérieur. Nous n'étions pas habillés chaudement non plus : l'habit religieux, avec une chemise de laine et des culottes courtes en toile comme sous-vêtements, une mante pour nos sorties en temps froid d'hiver. Rien pour nous protéger des morsures du froid. Les vents glacés nous traversaient littéralement et parfois nous gelaient dangereusement. Heureusement, durant mes études, les supérieurs se rendant compte de ce non-sens, ont accordé du linge plus chaud à tous les religieux, paletots, grands pantalons, casquettes, etc. À cette époque, notre province religieuse canadienne était encore relativement jeune. Les supérieurs étaient tous des Français ou des Canadiens qui avaient fait leur noviciat et leurs études en France. On se croyait obligé en conscience de suivre en pays froid le même régime de vie qu'on pouvait aisément supporter au sud de la France. On s'est vite aperçu qu'en pays froid, ce règlement était trop exigeant pour la santé. Je me rappelle un hiver où la fatigue faisait des ravages chez les étudiants, et en jetait quelques-uns dans de sérieuses dépressions.

Nos couvents vivaient dans une grande pauvreté. Un frère quêteur parcourait les campagnes pour quêter des légumes, et la ville pour les autres denrées nécessaires. Les gens étaient très charitables. Le frère était reçu avec beaucoup d'esprit de foi et de générosité. Les religieux n'avaient jamais un sou dans leurs poches, excepté pour des voyages lointains. Quand nous sortions pour aller chez le médecin ou chez le dentiste, nous n'avions rien d'autres que nos billets de tramways. Et tous les religieux étaient sur le même pied.

De mon temps, notre professeur de science n'était pas trop fort dans la matière qu'il avait à enseigner. Quand il était embêté par une question à laquelle il ne pouvait pas répondre, il s'en tirait avec cette solution rapide : « Le bon Dieu l'a voulu comme ça ». Quelques-uns cependant étaient diplômés des universités romaines. Quand j'étais étudiant, il n'était point question qu'un de nos jeunes religieux aillent poursuivre des études spécialisées dans une autre université que celles de Rome. À la fin de notre cours de théologie, je fus choisi avec un confrère, le père Marie-Antoine Painchaud, pour aller me spécialiser en théologie dans une université de la Ville Éternelle. Mais la guerre de 1939 empêcha la réalisation de ce projet.

\section{MON ORDINATION ET MON EUVRE À MONTRÉAL}

Je fus ordonné prêtre à la fin de ma troisième année de théologie. Vu que mon cousin, Paulo Boudreau, était ordonné à Chéticamp par son évêque, $\mathrm{M}^{\mathrm{gr}} \mathrm{Guy}$, de Gravelbourg, et que personne de ma famille n'aurait pu venir 
assister à mon ordination à Montréal, j'obtins le privilège d'être ordonné à Chéticamp le 11 juin 1938 en même temps que mon cousin.

On nous accordait deux ou trois semaines chez nous à l'occasion de notre ordination. Mais ma famille demeurait à cinq kilomètres de l'église et n'avait pas d'automobile. Il n'était guère pratique d'aller dire ma messe à chaque jour et de visiter la parenté en voiture à cheval. Un jour, je rencontrai le docteur Wilfred Poirier et lui fis part de mon embarras et de ma décision de raccourcir mes vacances pour cette raison. Il me dit : « Moi, j'ai une Austin comme deuxième voiture, mais elle n'a pas de freins, ni de lumières, ni de criard, ni de garde-boues. Si vous voulez vous en servir comme ça, elle est à vous pour le reste de vos vacances ". Avec une imprudence grave, le père Paulo et moi, nous fîmes cinq milles dans les montagnes de la Cabot Trail avec cette auto sans freins. Heureusement, c'est Paulo, plus expérimenté que moi à conduire une auto, qui tenait le volant. Dans les pentes raides, il laissait le moteur embrayé en première vitesse, pour monter comme pour descendre. Nous eûmes la frousse dans une montagne du Cap-Rouge que nous descendions, quand nous aperçûmes des ouvriers qui travaillaient sur le chemin et bloquaient le passage. Nous descendions lentement, mais sans freins, nous ne pouvions pas arrêter ; et nous n'avions pas de criard pour les avertir de libérer la voie. Heureusement, à la dernière minute, ils se sont écartés pour nous laisser passer.

\section{À deux doigts de la mort}

Il me restait une quatrième année de théologie à faire avant de recevoir la juridiction pour pouvoir confesser et exercer le ministère. À la fin de cette dernière année d'études, avant les examens, je fus atteint d'une crise d'appendicite. Et le docteur Trépanier, notre médecin de famille, qui n'avait pas le charisme du diagnostic, ne se rendit pas compte de la nature de la maladie. Il me laissa traîner jusqu'au jour où il me trouva blême comme un mort, en train de vomir. Alors pris de peur, il m'envoya d'urgence à l'hôpital l'HôtelDieu de Montréal. En me voyant le chirurgien, le docteur Paré, hurla presque : «Vite, à la salle d'opération ! Pourvu qu'il ne soit pas trop tard »! En m'ouvrant le ventre et en voyant une péritonite avancée, l'intestin percé, il dit : «Trop tard »! Mais ils se sont consultés, lui et ses deux assistants. Ils ont décidé de ne pas refermer la plaie et de laisser à l'air le bout d'intestin affecté. Dans tous nos couvents, on faisait des prières pour sauver le jeune prêtre que j'étais. Au bout de trois jours, l'air avait accompli le miracle et l'intestin était redevenu sain. On put le réintroduire dans mon ventre et refermer la plaie. 
J'avais terminé mes études avant de tomber malade, mais je n'avais pas passé les examens de fin d'année. Je le fis avec succès durant l'été et fut nommé professeur de philosophie de nos étudiants capucins. Mais, après avoir enseigné la logique durant six mois, je fus nommé professeur de théologie en remplacement du père Augustin Bolduc qui venait de mourir subitement. J'enseignai ainsi à la Chapelle de la Réparation de Montréal de 1940 à 1946.

\section{L'Ordre de Jacques Cartier}

En 1942, le père Marcellin, qui était supérieur du couvent et aumônier de la cellule locale de l'Ordre de Jacques Cartier, me présenta et me fit accepter comme son remplaçant dans cet ordre secret. Les membres se réunissaient une fois par mois en haut de la sacristie de notre chapelle. Quelques fois par année, avait lieu pour tout Montréal la cérémonie d'initiation des nouveaux membres. Elle avait lieu le plus souvent dans une immense salle de l'école Meilleure, où j'ai eu l'occasion de rencontrer l'élite canadienne-française de toute la région métropolitaine, comme le chanoine Lionel Groulx, le futur maire Jean Drapeau, Jacques Labrecque et combien d'autres.

Le dimanche de la fête du Christ-Roi, tous les membres de l'Ordre de la région de Montréal étaient invités à venir assister à une messe qu'on célébrait dans le galetas de notre couvent de la Chapelle de la Réparation. Comme ce grenier était $\mathrm{nu}$, la préparation pour le rendre convenable me demandait énormément de travail. Des centaines de membres y venaient. Je célébrais la messe et donnais le sermon de circonstance. La messe était suivie d'un lunch que quelques membres avaient préparé; ce qui donnait lieu à une fraternisation agréable.

\section{Contact avec les Acadiens de Montréal}

Durant ce temps, je maintenais des contacts réguliers avec les Acadiens de Montréal. Le 15 août, fête de l'Assomption, fête des Acadiens, je célébrais une messe pour eux à la Chapelle de la Réparation et donnais le sermon. La messe était suivie d'un pique-nique à l'extérieur, où chacun apportait son dîner.

\section{NOS CHANSONS D'ACADIE ET LE DÉBUT DE MA CARRIÈRE DE FOLKLORISTE}

En même temps que j'étais professeur à la Chapelle de la Réparation, mon cousin le père Daniel Boudreau, de Chéticamp, y était étudiant. Il avait en réserve des centaines de chansons de Chéticamp, dont plusieurs de notre 
commune grand-mère Résine Bourgeois. Nous avions conscience qu'elles étaient précieuses, mais nous ne savions pas trop quoi en faire. Or, à cette époque, l'abbé Charles-Émile Gadbois était célèbre pour ses recueils de la Bonne Chanson qu'il publiait. Je lui écrivis pour lui offrir nos chansons. Il ne daigna pas répondre à ma lettre.

Alors, on décida de commencer à les publier nous-mêmes, très modestement, seulement pour Chéticamp, que les chansons anglaises commençaient d'envahir par la radio. Le père Daniel, le musicien, transcrivait les mélodies; et moi l'éditeur, je tapais les textes et illustrais chaque chanson de dessins faits à la plume. Notre imprimerie consistait en une petite Multilith et des baudruches en plomb ou en zinc. Le travail était délicat et difficile. On ne pouvait pas toucher la baudruche avec nos doigts, car cela faisait des taches à l'imprimé; et on ne pouvait pas y faire de corrections si ce n'est en grattant la baudruche avec un couteau. Imaginez ! Écrire de la musique et exécuter des dessins dans des conditions pareilles !

Notre premier recueil parut en 1942 . Il contenait 25 chansons de folklore. Sa préparation nous avait mis en contact avec les folkloristes Thomas LeBlanc du journal $L$ 'Évangéline et surtout avec le célèbre Marius Barbeau. Les deux nous encouragèrent et Marius nous fit même une magnifique préface. Il nous invita à ses bureaux au Musée National de l'Homme à Ottawa où, muni d'une machine avec rouleaux en cire, il enregistra une foule de chansons chantées par le père Daniel et quelques-unes par moi. Il vint nous voir plusieurs fois au Lac Meech où nous avions nos vacances d'été.

Je fus déposer quelques exemplaires de notre recueil au grand magasin de musique Archambault de Montréal. L'abbé Gadbois les y découvrit. Il accourut aussitôt à la Chapelle de la Réparation pour obtenir le reste de notre répertoire. On lui répondit qu'il était trop tard; vu que nous avions commencé à les publier nous-mêmes, nous allions continuer. Il nous avoua qu'il cherchait depuis dix ans la chanson Partons la mer est belle que nous venions de publier. Il la publia peu après avec des variantes et, pour imposer sa version, la diffusa à grands renforts, dans un recueil, sur des feuilles volantes, sur des buvards, etc. C'est chez Archambault aussi que le chanteur Jacques Labrecque les découvrit. Il fit des milles en auto pour venir nous trouver au lac Killarney, près de Saint-Jovite, pour nous demander la permission de les chanter à la radio, au programme « le Réveil rural », dont il était le chanteur régulier.

En Acadie, nos recueils firent sensation. Ce fut une révélation, une surprise de voir que les Acadiens possédaient de tels trésors. Thomas LeBlanc en fit l'éloge dans L'Évangéline. Le père Breau, c.s.c. les fit chanter par sa chorale de l'Université Saint-Joseph de Memramcook et le père Médard Daigle par ses scouts. Les autres chorales, de Tracadie, du collège de Bathurst, les Alinos de Shédiac, et d'autres les ont chantées et endisquées. Édith Butler, Donat 
Lacroix et Angèle Arsenault ont commencé leur brillante carrière en chantant nos chansons.

Cette vogue de nos chansons, les contacts qu'elles m'avaient permis d'avoir avec Marius Barbeau, Luc Lacourcière, $M^{\mathrm{gr}}$ Félix-Antoine Savard, François Brassard et Carmen Roy me firent prendre conscience, à moi aussi, de l'importance et de la richesse du folklore acadien. Vers la fin des années 1950, Carmen Roy, qui avait succédé à Marius Barbeau au Musée de l'Homme à Ottawa, me prêta un magnétophone - ces machines commençaient à être sur le marché - et $\mathrm{M}^{\mathrm{gr}}$ Antoine Savard me donna cent dollars pour que je fasse des enregistrements de données folkloriques durant mes vacances d'été à Chéticamp. Puis à partir de 1959, Carmen Roy m'engagea quelques années, un mois par été, pour recueillir du folklore au Nouveau-Brunswick et surtout aux Îles de la Madeleine où je suis allé trois étés de suite, un mois durant, recueillir des centaines de chansons, des légendes, des contes et les traditions. Je devais envoyer ces enregistrements et leur transcription au Musée de l'Homme à Ottawa. Mais je gardais soigneusement pour moi une copie de tout.

\section{PRÉDICATEUR À CACOUNA}

En 1946, après avoir enseigné la théologie six ans, je fus nommé supérieur de notre couvent de Cacouna et prédicateur. Durant les trois années à ce poste, j'ai prêché des retraites paroissiales et des quarante-heures ; j'ai fait la visite canonique de nos nombreuses fraternités de l'Ordre séculier. J'ai aussi prêché des retraites dans des communautés religieuses, dans des écoles normales et des écoles ménagères. Cela m'a donné l'occasion de connaître beaucoup de paroisses et de prêtres, des religieuses et beaucoup de jeunes. $\grave{A}$ cette époque, je rêvais déjà de fonder une société d'histoire acadienne. Le 15 août 1948, j'avais convoqué à une réunion au collège de Bathurst un groupe de personnes qui auraient pu être intéressées. Mais peu après, je fus nommé curé à Ottawa et le projet s'éteignit là.

\section{MON EUVRE À OTTAWA}

En 1949, à 38 ans, je fus nommé curé de la grande paroisse de Saint-Françoisd'Assise d'Ottawa, qui avait été fondée par les capucins en 1890. Elle comptait au-delà de mille familles. Je n'avais jamais fait de ministère en paroisse, si ce n'est y prêcher des retraites. À mon arrivée, j'avais cinq vicaires, tous plus âgés que moi. Ces vicaires ont été charitables et $m$ 'ont initié à ce ministère tout nouveau pour moi. 
Emballé par le succès des chansons d'Acadie que nous avions publiées, je voulus faire goûter la beauté et la richesse de la chanson française aux élèves de nos écoles et à leurs parents. Je demandai à chaque classe de nos quatre écoles de préparer un chant, à être chanté et mimé par tous les élèves de la classe en vue d'un premier festival paroissial. Il eut lieu le 23 avril 1950 et fut une grande réussite.

\section{Une colonie de vacances}

En octobre 1950, M. Marcel Demers, gérant de la Caisse populaire, m'annonce qu'une colonie de vacances, Notre-Dame-de-la-Joie, est à vendre. Elle est située à Beechgrove, dans les montagnes, sur la route entre Masham et Luskville. Le propriétaire accepta de la vendre pour $10000 \$$. La première année fut difficile. La température fut maussade tout l'été et nous manquions d'expérience. Au mois d'août, je visitai d'autres camps d'enfants et, de là, j'adaptai au nôtre ce que j'y avais trouvé de meilleur.

Nous recevions des subventions des clubs sociaux et même du gouvernement du Québec, qui nous permettaient d'accepter gratuitement les enfants pauvres de la paroisse et plusieurs de Hull, ceux qui en avaient le plus besoin. On pouvait prendre jusqu'à 120 enfants à la fois. Les demandes venaient de partout, de l'Ontario et même de Montréal et de Québec. La bonne renommée de cette colonie de vacances constituait sa meilleure publicité.

\section{Problème de voiture et fin de mon mandat}

À cette époque, les capucins n'avaient pas d'autos et il ne leur était pas permis d'en avoir. Or, j'avais absolument besoin d'une voiture, d'une camionnette, pour desservir la colonie de vacances, située à trente milles d'Ottawa. Pour contourner la défense, je fis acheter une vieille camionnette au nom des zouaves de la paroisse. Mais le père provincial du temps et ses conseillers s'opposèrent même à ça. Il était pourtant impossible de faire fonctionner la colonie de vacances sans voiture. Le cas est devenu un problème provincial. On vint me trouver pour me dire qu'on voulait m'élire comme un des conseillers du père provincial, mais à condition que je renonce à la voiture. Je les envoyai promener. J'exposai calmement la nécessité d'une voiture pour la colonie de vacances, mais aussi pour le ministère moderne, visite des malades à l'hôpital une fois par semaine, commissions multiples ici et là, etc. Mais le conseil provincial continua de s'opposer farouchement à notre voiture. Et ce fut probablement une des principales raisons pour me retirer ma cure en 1957, en plein milieu d'un mandat triennal, pour me nommer professeur de théologie morale. Le dimanche où l'on annonça en chaire mon changement, les gens pleuraient et n'y comprenaient rien. La paroisse était 
plus vivante qu'elle ne l'avait jamais été. On aurait été moins surpris, je crois, si on avait annoncé que j'étais nommé évêque.

Dans la province des capucins, beaucoup crurent que $j$ 'avais dû faire un mauvais coup ou avoir eu une mauvaise conduite. Le supérieur du couvent d'Ottawa était un de ceux-là. En plus d'être curé, j'avais un rayonnement important chez les Franco-Ontariens comme un des directeurs de l'Association d'éducation franco-ontarienne, où j'ai beaucoup travaillé avec le président, l'avocat Gaston Vincent, aussi dans la Fédération des foyers-écoles dont j'étais l'un des directeurs-fondateurs. Ces groupes et d'autres voulaient que je continue à œuvrer avec eux. Or, mon supérieur était très réticent à me laisser sortir, au point que je dus cesser mes activités dans ces groupes. C'est alors qu'une délégation de Franco-Ontariens allèrent à mon insu trouver le père provincial pour lui expliquer l'importance de mon travail pour la cause francoontarienne et lui demander la permission pour moi de continuer à œuvrer avec eux.

Je suis resté un an et demi à Ottawa à enseigner la théologie morale, et continuai à être actif dans les mouvements franco-ontariens. Mon meilleur exploit fut à un congrès, tenu à Ottawa, par les membres de l'Association d'éducation et de la société Saint-Jean-Baptiste franco-ontariennes. L'Association avait toujours été considérée et avait agi comme la société mère des Franco-Ontariens. Toutes les autres sociétés francophones lui étaient affiliées.

\section{L'Ordre de Jacques Cartier}

À Ottawa, je continuai à œuvrer dans l'Ordre de Jacques Cartier. Le siège social se trouvait dans cette ville. En 1957, le secrétaire général, $M^{e}$ Léopold Allard, m'invita à faire avec lui la visite de toutes les commanderies des provinces maritimes. En plus de visiter les commanderies déjà existantes, on en fonda d'autres, en particulier à Sydney et à Chéticamp. $\mathrm{M}^{\mathrm{e}}$ Allard ne se rendit pas à Chéticamp. Rendu à Sydney, il n'en pouvait plus de fatigue et il m'envoya seul fonder à Chéticamp. Le soir de la réunion de fondation, je prononce un discours pour démontrer le bien que l'Ordre accomplit partout et qu'il pourrait accomplir à Chéticamp pour la sauvegarde de la langue française, sa devise étant : Langue et foi. Puis on demande au père curé de dire le mot de la fin. Son discours fut une descente contre l'établissement d'une cellule de l'Ordre à Chéticamp, affirmant que la paroisse n'avait pas besoin de ça. J'étais inquiet de la tournure des événements, quand le président, Amédée Boudreau (à Placide) prit la parole et eut le cran de dire : «Père Comeau, vous ferez ce que vous voudrez. Vous pouvez en faire partie ou non, mais nous fondons une cellule de l'Ordre de Jacques Cartier à Chéticamp et elle va y œuvrer avec vous ou sans vous ». 
EN ACADIE

\section{À Bathurst}

En 1958, je fus nommé supérieur et prédicateur à notre couvent de ValléeLourdes à Bathurst. Pendant un an, j'ai prêché dans plusieurs paroisses de ce diocèse. J'ai même accompagné l'évêque, $\mathrm{M}^{\mathrm{gr}}$ Camille LeBlanc, dans une tournée de confirmation. J'ai trouvé les prêtres des paroisses de Bathurst très sympathiques. Durant l'espace d'une seule année où je suis demeuré à Bathurst, je me suis fais plusieurs bons amis parmi eux.

Je recueillais depuis trente ans de la documentation pour mon livre sur l'histoire de Chéticamp. Je m'étais mis à l'écrire depuis que je n'étais plus curé et $\mathrm{j}$ 'en terminai la rédaction à Bathurst. Une religieuse des sœurs Hospitalières en tapa le texte et mon ami, Éphrem Boudreau d'Aylmer, près d'Ottawa, en fit la révision et les corrections. Ses corrections de mon premier volume me furent une véritable leçon d'écriture.

\section{À Moncton}

J'avais été envoyé à Bathurst par mes supérieurs en vue d'étudier la possibilité d'ériger un juvénat pour les jeunes Acadiens désireux de devenir capucins. Notre père provincial, le père Arthur Bolduc, vint visiter Moncton avec moi. On prit une option sur un beau grand terrain à Saint-Anselme, qui donnait sur la rivière Petitcodiac. Mais on décida d'acheter pour le moment une grande maison à deux loyers à 277-279 rue Dominion, Moncton. Au lieu de bâtir un collège et d'y mettre des professeurs, on adopta une formule qui parut extraordinaire à l'époque, celle d'envoyer nos juvénistes pour leurs cours à l'externat classique L'Assomption situé tout près et dirigé par des prêtres séculiers. Il semble que c'est la première fois au Canada qu'une communauté religieuse confiait l'étude de ses juvénistes à d'autres qu'à leurs propres professeurs. Aujourd'hui, cela nous fait rire.

Je fus nommé supérieur à notre nouvelle résidence de Moncton, où j'arrivai au mois de juillet 1959. La maison était vide, sans même une chaise pour s'asseoir. Un ami de Chéticamp, Louis-Philippe Chiasson, qui était passé me voir, fut si frappé de mon dénuement qu'il voulait faire une collecte pour moi de retour chez lui. Je l'en dissuadai, l'assurant que les meubles ne tarderaient pas à arriver de Montréal.

Pour en finir avec notre juvénat, disons que nous avons eu jusqu'à vingt élèves à la fois dans notre maison. C'était bien organisé et tout fonctionnait bien. Mais avec l'ouverture de l'Université de Moncton, l'externat L'Assomption a fermé ses portes et, la crise qui a suivi le Concile aidant, nous avons dû fermer notre juvénat. 


\section{Mon livre de Chéticamp}

Luc Lacoucière de l'Université Laval m'avait offert de publier mon livre de Chéticamp dans sa collection Les Archives de folklore. Mais selon sa lenteur habituelle, il eut le manuscrit pendant plus de deux ans sans le publier. J'en eus assez d'attendre. Je suis allé chercher le manuscrit, décidé de le publier moi-même à compte d'auteur. M. Lacourcière me remit aussi la magnifique préface qu'il avait composée pour mon livre.

Comme je prévoyais publier d'autres livres, je décidai de fonder une maison d'édition, Les Éditions des Aboiteaux. Si modeste fût-elle, ce fut la première maison d'édition française en Acadie. Je publiai donc mon livre Chéticamp, histoire et traditions acadiennes en 1961. Il reçut des louanges extraordinaires auxquelles je ne $m$ 'attendais guère. Le succès de ce livre est dû au fait qu'il est une des premières monographies paroissiales au Canada qui, en plus des événements historiques, décrit abondamment la vie des gens.

\section{En quête de données folkloriques}

J'avais déjà fait, pour le Musée de l'Homme, plusieurs enregistrements sur magnétophone en Nouvelle-Écosse, à Chéticamp, Saint-Joseph-du-Moine, et au Nouveau-Brunswick : à Néguac, Paquetville, Robertville, Adamsville et, surtout, à Acadieville et Rogersville. En 1961, je proposai au Musée de l'Homme d'aller aux Îles-de-la-Madeleine. Ce fut accepté.

Je n'oublierai jamais ma première traversée aux Îles. À cette époque, on prenait le bateau à Pictou. L'équipage était composé entièrement de Madelinots. Le trajet se faisait de nuit. À minuit, je montai à la cabine du pilote. Un bon Madelinot, un M. Robichaud d'un âge avancé était à la roue. La mer était calme et la lune s'y miroitait de milliers de reflets. Ce monsieur me reçut gentiment et la conversation s'entama. Je lui dis que j'allais aux Îles recueillir du folklore, des chansons, des contes, des légendes et des traditions. Je lui demandai s'il connaissait des chansons des îles. Et pour la première fois, sur la mer, sur l'heure de minuit, $j$ 'entendis chanter par ce vieux pilote la chanson des Îles : «J'aime ce petit coin de terre perdu là-bas aux grandes eaux $»$. Ce fut très émouvant.

Je ne connaissais personne aux Îles. Mais j'avais écrit à $\mathrm{M}^{\mathrm{gr}}$ André Arsenault, curé de Lavernière et vicaire général des Îles, pour lui demander l'hospitalité. Arrivé au presbytère, je fus reçu cordialement; mais, après lui avoir expliqué le but de mon voyage, Monseigneur m'affirma qu'il doutait fort que je trouve aux Îles quoi que ce soit du matériel que je venais y chercher. Il me conseilla cependant de m'adresser à Avila LeBlanc de Gros-Cap, un célibataire presque aveugle. Ce conseil fut des plus précieux. Avila était l'homme à contacter. Violoneux lui-même, il possédait un riche répertoire 
de chansons, d'histoires et de légendes des îles. En plus, il connaissait bien tous les bons informateurs dont $\mathrm{j}$ 'aurais besoin et accepta avec plaisir de m'accompagner chez eux.

On m'avait averti d'être prudent dans mon approche des informateurs. Aux Îles, on se méfiait des enquêteurs étrangers. Des gens de l'Office national du film et de Radio-Canada y étaient venus et avaient filmé tout ce qu'il y avait de plus minable qu'ils avaient présenté ensuite comme représentatif des Îles. Dans leur colère, les Madelinots avaient jeté beaucoup de discrédit sur leur culture populaire, considérée des vieilleries.

Avila était aimé et respecté de tous; sa présence avec moi m'assurait déjà un accueil bienveillant. En plus, le fait que j'étais prêtre et aussi natif de Chéticamp m'aida beaucoup. Pour les Madelinots, les gens de Chéticamp sont les frères acadiens les plus proches, avec qui on a toujours eu des relations amicales. Aux Îles, pour quelqu'un de Chéticamp, les portes et les cœurs sont toujours largement ouverts. Au commencement en effet, les gens furent réticents; mais il me fallut peu de temps pour les convaincre de la beauté de leur folklore, de ce trésor qui leur appartenait, qu'il était important de sauver et même de faire valoir. La collaboration fut vite cordiale et entière.

Durant mon séjour aux Îles, tout le mois de juillet durant trois années de suite, je travaillais fort tous les jours. L'après-midi, je me rendais chez certaines familles où il y avait des personnes âgées. Le soir, j'allais dans d'autres maisons, qui se remplissaient de personnes intéressées. J'enregistrais des contes et des légendes l'après-midi, et des chansons le soir, jusqu'à minuit. Le lendemain matin, je faisais entendre une partie de ma cueillette à $\mathrm{M}^{\mathrm{gr}}$ Arsenault qui n'en revenait pas de la richesse de mes trouvailles. J'enregistrai aussi des violoneux, d'abord Avila LeBlanc lui-même, un Longuépée de Pointe-aux-Loups, aussi et surtout des Lapierre des Caps-ouest.

\section{La Société historique acadienne}

En 1960, je ne connaissais pas beaucoup de monde à Moncton où je venais d'arriver. Or, je voulais fonder tout de suite une société historique que je considérais comme une nécessité urgente pour les Acadiens. Je connaissais bien Émery LeBlanc rédacteur en chef de L'Évangéline. Je fus lui faire part de mon projet et lui demander s'il voulait m'aider à fonder cette société. Il se montra enthousiaste et me dit : «Si nous pouvions gagner le père Clément Cormier à l'idée, nous aurions beaucoup plus de chance de réussir ». Le père Cormier fut approché et lui aussi se montra enthousiaste. En effet, il mijotait le même projet depuis plusieurs années, comme le prouve son mémoire à la Commission Massey (1949-1951) où il recommandait la fondation d'un Institut d'histoire d'Acadie avec une Société historique et une revue. Il se mit tout de suite à écrire la constitution de cette société. Il accepta d'en être 
le premier président et Émery fut le premier secrétaire. J'insistai pour qu'on publie une revue immédiatement. Pour le premier numéro on décida de solliciter des annonces, ce dont se chargea Émery. Quant à moi, je pris la direction des Cahiers dès 1964. Émery n'avait publié que trois numéros en tout, mais ils étaient volumineux. La première année, je montai le nombre à trois, puis à quatre toutes les années suivantes. J'ai gardé la responsabilité des Cahiers durant dix ans pour une première fois, puis deux autres années à partir de 1984. Le 10 mai 1992, je fus nommé conseiller à vie de la Société historique.

\section{Les soirées acadiennes}

À partir de 1960, Léo Cormier de Radio-Canada, qui connaissait nos Chansons d'Acadie pour les avoir chantées avec la chorale du père Brault du Collège Saint-Joseph, organisa des soirées acadiennes pour la radio. L'équipe de Radio-Canada se transportait dans une famille acadienne de la paroisse où se tenait la soirée. On enregistrait des violoneux, des chansons de folklore, des histoires et des danses. Une quarantaine de soirées tenues dans les trois provinces Maritimes furent enregistrées durant l'espace de trois ans. Je faisais partie de l'équipe et commençais le programme en donnant l'historique de la paroisse visitée. Ces soirées étaient très goûtées des auditeurs qui auraient voulu qu'elles continuent.

\section{Fin de mes prédications}

À mon arrivée en Acadie en 1959, j'avais continué le ministère de la prédication commencé à Cacouna en 1946. Je prêchais surtout des retraites paroissiales et des quarante heures dans la province, mais aussi au Québec durant l'avent et le carême. Il semble que je réussissais assez bien. Je préparais bien mes sermons au point de pouvoir improviser facilement. Je n'oublierai jamais une retraite que j'ai prêchée à Acadieville (N.-B.), où la communication avec l'auditoire fut particulièrement intense et les grâces abondantes dans la paix et la joie. Je me suis toujours efforcé d'éviter le ton prêchi prêcha. Je parlais aux gens, n'ayant pas peur de les dérider de temps en temps.

Le 7 octobre 1962, j'arrivais à Saint-Eustache (Québec) pour y prêcher une retraite paroissiale. Je me rappelle la difficulté que $\mathrm{j}$ 'avais eue à me rendre de l'autobus au presbytère avec ma valise. Depuis plusieurs mois, je sentais une fatigue anormale. Mais c'est lors de la retraite que nous prêchions ensemble plusieurs capucins à Saint-Jean-d'Iberville, en 1963, que le malaise arriva à son point culminant. Le docteur Florent Thébert, frère d'un vicaire de la paroisse, étant venu en visite, m'examina et crut bon de m'amener à 
l'hôpital Rosemont de Montréal pour examen. Les radiographies indiquaient des taches sur un poumon. Le docteur Lafortune m'opéra et m'enleva deux lobes du poumon droit.

\section{Un voyage avec des membres de la Commission Laurendeau-Dunton}

La Commission Laurendeau-Dunton sur le bilinguisme avait été créée par le premier ministre canadien Lester Pearson. Cette commission avait demandé au père René Beaudry, c.s.c. qui était archiviste pour le Canada à Paris, de préparer une étude sur les Acadiens de 1850 à nos jours. Le père Beaudry avait consacré tout le temps de ses vacances au Canada à faire les recherches nécessaires. Mais il n'avait rien écrit. Il m'invita à aller avec lui à Paris. Il payait mes dépenses de voyage et mille dollars pour trois mois de travail. C'était peu comme salaire, mais j'acceptai. Je pris l'avion le 8 juin 1965 à Moncton, avec le père Beaudry. Je décidai d'accomplir mon travail d'abord, avant de faire toute visite de Paris. Je complétai la documentation du père Beaudry qui n'avait rien sur la culture des Acadiens, ni sur leurs problèmes avec la hiérarchie religieuse anglophone, etc. J'écrivis la grande partie des deux volumes que le père Beaudry put livrer en temps et en son nom à la Commission.

\section{L'île de Shippagan, anecdotes, tours et légendes}

Au début de l'année 1967, j'ai publié L'Île de Shippagan, anecdotes, tours et légendes, dont le contenu avait été préparé à ma suggestion par mon ami Francis Savoie de Montréal, natif de Lamèque. J'avais encore en main le brouillon qu'il m'avait envoyé, lorsque j'appris son décès. Je retournai le manuscrit à son épouse en lui disant qu'il avait de la valeur, mais qu'elle devait trouver quelqu'un de compétent pour le récrire. Personne n'ayant accepté d'entreprendre ce travail, elle me l'avait renvoyé me priant d'accomplir cette tâche pour mon ami Francis. Ce que j'ai fait. Pendant un an, j'ai récrit ce texte en entier et je l'ai publié aux Éditions des Aboiteaux au nom de Francis Savoie, avec des illustrations de Claude Roussel. J'indique dans la préface la part que j'y ai apportée; mais j'aurais pu et peut-être dû mettre nos deux noms comme co-auteurs.

\section{La cause française en Nouvelle-Écosse}

Le 10 juin 1969, nous publiions dans L'Évangéline, Alexandre Boudreau et moi, un article virulent sur la menace à la langue française qui planait sur l'école de Chéticamp. Après avoir souligné le rôle salvifique joué par les religieuses Filles de Jésus pour la cause française dans le passé, nous les 
accusions d'être à cette époque des fauteurs d'anglicisation. Invité par les Dames chrétiennes de l'endroit et par la paroisse, je me rendis à Chéticamp le dimanche du 15 août, où à l'église, après la messe, je prononçai un discours sur l'importance de la langue française et les moyens à prendre pour la conserver et la promouvoir.

\section{Le journal $L$ 'Évangéline}

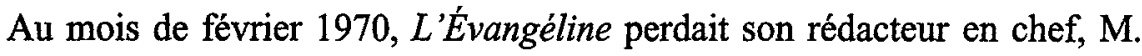
Bernard Poirier, qui acceptait un emploi du gouvernement provincial à Frédéricton. N'ayant pas de remplaçant, les autorités du journal réunissent un comité afin de trouver une formule de rechange. La trouvaille fut que l'on me chargea de la responsabilité de fournir des éditoriaux, quitte à en écrire moi-même et à en solliciter d'un groupe que je verrais à former à travers la province. Nous avons exigé que nos éditoriaux ne soient pas signés, personne n'ayant le temps de répondre aux lettres que ces écrits pourraient susciter.

De décembre 1970 à mai 1972, j'ai aussi écrit dans le journal L'Évangéline une chronique hebdomadaire en parler acadien, intitulée « Le coin à Piquine ». Je le faisais pour m'amuser et aussi pour donner un exemple du véritable parler acadien, en réaction contre certains écrits qu'on présente comme du parler acadien, alors que ce n'est que du charabia.

\section{Au Centre d'études acadiennes}

Au début de mars 1966, le père Clément Cormier, recteur de l'Université de Moncton, m'invita à devenir l'archiviste du Centre d'études acadiennes. Dans la construction de la bibliothèque sur le nouveau campus, il avait réservé des locaux pour le Centre. Mais en 1966, seul Ronald LeBlanc y travaillait et, en plus, il était un peu l'homme à tout faire dans la grande bibliothèque ellemême. Vu que mes poumons ne me permettaient plus de prêcher, j'acceptai l'invitation et commençai à travailler le lundi 14 mars. Tout de suite, je m'intéressai avec Ronald LeBlanc à monter le Centre de toute la documentation possible sur l'Acadie et les Acadiens. Et quand le père Clément en fut nommé directeur un 1968, je devins comme son bras droit. Comme il avait beaucoup de crédit auprès du recteur qui lui avait succédé, il obtenait facilement du personnel et des fonds au profit du Centre. C'est ainsi que nous y avons fondé le département de folklore et engagé l'ethnomusicologue Charlotte Cormier, et celui de généalogie avec le père Hector Hébert, s.j.

Le père Clément obtint du Conseil des arts du Canada une subvention en vue de préparer des instruments de recherche. Elle servit à produire le premier tome : Inventaire général des sources documentaires sur les Acadiens. Je fis l'inventaire des archives du Centre pour cette œuvre et, à la fin, comme le 
père Clément était malade, je dus prendre la responsabilité de toute l'œuvre, qui ne fut publiée d'ailleurs qu'en 1975, alors que je l'avais remplacé en tant que directeur du Centre. Le 3 janvier 1976, j'avais 65 ans et selon les règlements de l'Université, je devais prendre ma retraite en tant que directeur du Centre d'études acadiennes. Je crois que je peux affirmer sans vantardise que j'ai été le grand promoteur du développement du Centre d'études acadiennes pour réussir à en faire un Centre unique au monde de documentation sur l'Acadie et les Acadiens. Mes successeurs ont continué dans le même sens.

\section{Conseiller des étudiants et aide aux écrivains en herbe}

Alors que j'étais archiviste ou directeur du Centre d'études acadiennes, j'ai toujours cherché à aider les étudiants dans leurs études, dans leurs recherches et dans le choix de leurs thèses. Même à ma retraite, $\mathrm{j}$ 'ai continué à encourager et à aider beaucoup d'écrivains en herbe, les orientant, corrigeant leurs textes. Que d'articles écrits pour la revue de la Société historique acadienne que j'ai dû corriger et retravailler !

\section{MA VIE SPIRITUELLE}

Tout ce qui précède n'est que la description de mes activités secondaires, car, le plus important dans ma vie, c'est le côté spirituel et sacerdotal. Aussi, je crois qu'il convient que j'en dise quelques mots. J'ai raconté au début ma dévotion au saint sacrement et à Marie durant mon enfance, due à la lecture de la vie de saint Gérard Majella. Naturellement, au juvénat, au noviciat et durant mes études en préparation au sacerdoce, notre piété était nourrie de conférences et de lectures spirituelles. J'ai continué comme prêtre à alimenter mon âme de telles lectures et d'oraisons. J'ai beaucoup lu de livres de spiritualité; mais ceux qui m'ont fait et me font encore plus de bien, ce sont les vies des saints. Leur exemple concret est un stimulant qui nous inspire une plus grande générosité à répondre aux inspirations de la grâce. Les écrits des mystiques, écrits par obéissance à leurs supérieurs ou à leur directeur de conscience, racontant l'œuvre du Seigneur en eux ou en elles, ont maintenu chez moi une foi vive et une grande piété. Comme directeur spirituel de futurs prêtres, je favorisais chez mes dirigés cette piété où toute compromission, toute illusion étaient bannies : d'abord garder l'état de grâce, confessions régulières, lectures spirituelles assidues et être à l'écoute des inspirations ou des désirs du Seigneur et y répondre généreusement.

Dans mes cours de théologie, contrairement au manuel qui n'avait qu'un petit paragraphe sur la grâce sanctifiante, je développais ce sujet pendant plusieurs cours, parce que c'est la merveille des merveilles, le don de Dieu, la vie divine en nous, qui nous fait enfants de Dieu, d'autres Christs, puisque 
nous avons la même vie divine que lui. Nous ne faisons qu'un avec lui (Corps mystique), le «ce que vous faites au plus petit des miens, c'est à moi " se comprend, le mérite infini de nos vies (l'œil de l'homme n'a jamais vu, ni son oreille entendu, ni son cœur compris ce que Dieu réserve...), etc. Plus j'avance dans la vie, plus je vis ma messe dans la foi et plus Jésus me fait comprendre les merveilles que Dieu fait pour nous par amour.

Père Anselme Chiasson, cap. 